\title{
Viscosity calculated in simulations of strongly-coupled dusty plasmas with gas friction
}

\author{
Yan Feng* J. Goree, and Bin Liu \\ Department of Physics and Astronomy, The University of Iowa, Iowa City, Iowa 52242, USA
}

(Dated: November 7, 2018)

\begin{abstract}
A two-dimensional strongly-coupled dusty plasma is modeled using Langevin and frictionless molecular dynamical simulations. The static viscosity $\eta$ and the wave-number-dependent viscosity $\eta(k)$ are calculated from the microscopic shear in the random motion of particles. A recently developed method of calculating the wave-number-dependent viscosity $\eta(k)$ is validated by comparing the results of $\eta(k)$ from the two simulations. It is also verified that the Green-Kubo relation can still yield an accurate measure of the static viscosity $\eta$ in the presence of a modest level of friction as in dusty plasma experiments.
\end{abstract}

PACS numbers: 52.27.Lw, 52.27.Gr, 66.20.-d, 83.60.Bc

\section{INTRODUCTION}

Strongly-coupled plasma is a collection of free charged particles where the Coulomb interaction with nearest neighbors is so strong that particles do not easily move past one another. A widely used criterion to determine whether a plasma is strongly coupled is $\Gamma>1$ [1, 2], where $\Gamma$ is defined as the ratio of the potential energy between neighboring particles and the kinetic energy. When $\Gamma>1$, particles move slowly and are trapped by a cage consisting of a few nearby particles. If they escape the cages gradually, particles in a strongly-coupled plasma can flow, much like a liquid [3]. However, if $\Gamma \gg 10^{2}$, nearby particles that form a cage move so little that a particle inside the cage can seldom escape the cage; this condition is like molecules in a solid [4, 5]. If a shearing stress is applied, cages in a solid are elastically deformed but can restore to their previous state, whereas cages in a liquid are disrupted and a viscous flow can develop.

One type of strongly-coupled plasma is dusty plasma formed in the laboratory. A dusty plasma consists of four constituents: micron-size particles of solid matter (dust particles), electrons, ions, and neutral gas atoms [6 8 ]. The dust particles are strongly coupled amongst themselves due to a large interparticle potential energy provided by a large particle charge [9, 10]. Several schemes have been used to confine charged dust particles using natural electric field inside a plasma. One of these schemes makes use of a radio-frequency plasma [11, 12], with a horizontal electrode that provides a sheath electric field that can confine and levitate dust particles in a cloud with only a few horizontal layers. If experimenters introduce only a limited number of dust particles, they can settle into just a single layer [9]. In these single-layer clouds, dust particles have negligible vertical motion, so that the cloud of dust particles is often described as a two-dimensional (2D) system [9, 13 16]. In this $2 \mathrm{D}$ cloud, the interaction between dust particles is a repulsive Yukawa potential [17]. Due to the large length scale and the slow time scales [8], dusty plasmas allow video microscopy to track individual particle motion [18]. In dusty plasma experiments, elasticity in solids [14] and viscosity in liquids [16] has been observed and studied. However, strongly-coupled plasmas cannot always be classified as purely elastic or purely viscous.

Dust particles experience several forces in the experiments. The electric force provides strong coupling amongst the dust particles as well as the levitation and confinement. Gas friction, due to dust particles moving relative to the rarefied gas, is the primary energy loss mechanism. The gas is usually so rarefied that it represents only a small portion of the mass of the dusty plasma. Gas represents $<10 \%$ of the mass of dust in a $3 \mathrm{D}$ dusty plasma experiment at $400 \mathrm{mTorr}$ [19], while $2 \mathrm{D}$ experiments have even less gas, with a pressure $<20$ mTorr [9, 20, 21]. There is an ion drag force due to a steady flow of ions, arising from the same dc electric fields that provide levitation and confinement of dust particles. This ion drag force is parallel to the ion flow. Finally, in some experiments, laser radiation pressure forces are used to accelerate dust particles, for example to create macroscopic flows [12, 16, 21, 22] or simply raise the kinetic temperature of the dust particles without causing a macroscopic flow $[9,20,23,24]$. This kind of laser heating method is one of several ways that experimenters can control $\Gamma$ so that the cloud of dust particles behaves like a liquid or solid [13, 24 27].

We assume that the Coulomb interaction amongst charged dust particles is the dominant mechanism for viscosity in laboratory dusty plasma experiments. Viscous transport of momentum occurs when the dust particles moving relative to one another in a shearing motion collide, causing some of their momentum to be transferred across the flow. We expect that collisions involving gas atoms will contribute less to the viscosity. Although the force of gas friction is effective in diminishing momentum of dust particles in the direction of their motion, there are two reasons it has little effect in transferring momentum across a flow of dust particles. First, the gas is rarefied so that it can carry much less momentum than a viscous solvent in a colloidal suspension 28, 29], for example. Second, in a 2D experiment like [20], a gas 
atom that is struck by a dust particle is usually knocked into a direction out of the dust layer, so that there is little opportunity for a dust particle to push another dust particle indirectly through collisions with a gas atom.

Here, we will refer to the viscosity as the static viscosity $\eta$ to distinguish it from viscoelasticity. In the literature of dusty plasmas, the static viscosity $\eta$ has been measured experimentally [16] and quantified in simulations [30 33. There are two ways to quantify the static viscosity. If there is a macroscopic velocity shear, the static viscosity can be calculated from the velocity flow profile 12,16 , 32, 33]. On the other hand, if there is no macroscopic velocity shear, the microscopic shear associated with the random motion of particles can be used to calculate the static viscosity using the Green-Kubo relation [31, 33, 34].

Viscoelasticity is a property of materials that exhibit both liquid-like viscous and solid-like elastic characteristics 35]. Most materials in reality are viscoelastic, such as wood, synthetic polymers, and human tissue 35. Viscous effects correspond to energy dissipation, while elastic effects corresponds to energy storage. In general, liquids exhibit mostly viscous effects at large spatial and temporal scales, but they exhibit some elastic effects at small spatial and temporal scales [36].

To characterize viscoelasticity quantitatively, it is common to use either the frequency-dependent viscosity $\eta(\omega)$ or the wave-number-dependent viscosity $\eta(k)$. The latter characterizes materials at different length scales, and was introduced by theorists performing simulations [37-40]. The static viscosity $\eta$ is the hydrodynamic limit of the wave-number-dependent viscosity $\eta(k)$ when $k \rightarrow 0$. In considering this limit, the relevant characteristic length scale for $k$ is the interparticle distance, which is often measured as the lattice constant $b$ of a perfect crystal.

Viscoelasticity of strongly-coupled plasmas has been studied theoretically [41 43] and experimentally [20, 44, 45]. The few experiments that have been reported for viscoelasticity of dusty plasma include a descriptive presentation [44] and a characterization using a correlation function of the microscopic motion of dust particles 45 .

In our recent 2D experiment 20], a single horizontal layer of electrically charged dust particles was levitated in a glow-discharge plasma. The kinetic temperature of the dust cloud was raised by laser heating [20, 24]. Viewing from above, we recorded movies of particle motion, then calculated particle positions and tracked them to calculate their velocities. Based on the trajectories of particles, the wave-number-dependent viscosity $\eta(k)$ of the 2D dusty plasma was quantified using an expression we derived that accounts for gas friction.

In simulations, the viscoelasticity of both $2 \mathrm{D}[20]$ and 3D strongly-coupled plasmas [43] have been studied recently. In this paper, we carry out further simulations for two purposes: to validate the $\eta(k)$ calculation method taking into account gas friction, as presented in [20], and to assess the accuracy of the Green-Kubo relation for dusty plasmas with a modest level of gas friction.

Simulations of strongly-coupled plasmas usually use the molecular dynamical (MD) method [20, 43]. Each particle is tracked individually, unlike the case of particlein-cell (PIC) simulations, where aggregations of particles are simulated by a hypothetical super-particle [7]. Tracking individual particles is suitable because otherwise the dominant effects of strong particle-particle Coulomb interactions would be lost. Another difference is that in MD simulations, as compared to PIC simulations, Poisson's equation is not solved. The only equation that is solved is the equation of motion for each particle, which is integrated to track particle trajectories. The result of the MD simulation is a record of all particle positions and velocities, which is the same kind of data that are produced in dusty plasma experiments. The interparticle interaction that is assumed in MD simulations of strongly-coupled dusty plasmas is a repulsive Yukawa potential [17],

$$
\phi_{i, j}=Q^{2}\left(4 \pi \epsilon_{0} r_{i, j}\right)^{-1} \exp \left(-r_{i, j} / \lambda_{D}\right),
$$

where $Q$ is the charge on dust particles, $\lambda_{D}$ is the Debye length, and $r_{i, j}$ is the distance between the $i$ th and $j$ th particles.

We list here additional parameters for the dusty plasma cloud. Because the dust cloud is $2 \mathrm{D}$, we use an areal number density $n$ and an areal mass density $\rho=m n$ for the cloud, where $m$ is the dust particle mass. We note that while the units for mass density and viscosity are different in $2 \mathrm{D}$ and $3 \mathrm{D}$, the units are the same for the kinematic viscosity [16], $\eta / \rho$. Distances between dust particles are characterized by both the lattice constant $b$ for a crystal or the 2D Wigner-Seitz radius $a=(n \pi)^{-1 / 2}$ [46]. Time scales for collective motion are characterized by the nominal $2 \mathrm{D}$ dusty plasma frequency [46] $\omega_{p d}=\left(Q^{2} / 2 \pi \epsilon_{0} m a^{3}\right)^{1 / 2}$. Gas friction is characterized by the damping rate $\nu_{f}$, which is the ratio of the gas friction force and the dust particle's momentum.

We will discuss how to calculate $\eta$ and $\eta(k)$ in Sec. II. In Sec. III, we will discuss our two MD simulation methods, Langevin and frictionless. In Sec. IV, we will report new simulation data for $\eta(k)$ of $2 \mathrm{D}$ strongly-coupled dusty plasmas. We will validate our analysis method [20] for calculating $\eta(k)$ in 2D strongly-coupled plasma with gas friction. We will also test the accuracy of the GreenKubo relation with a modest level of gas friction as in our experiment. 


\section{METHODS FOR CHARACTERIZING VISCOSITY}

\section{A. Static viscosity $\eta$}

The Green-Kubo relation is widely used for calculating the static viscosity $\eta$, based on the random motion of particles. This method is used when there is no macroscopic velocity shear. The Green-Kubo approach assumes linear microscopic fluctuations and equilibrium fields in the system 31]. The assumptions of this approach are similar to those for the fluctuation-dissipation theorem [47, 48]. Previously, the Green-Kubo relation was generally used with data from frictionless simulations $30,31,33,34,43$. To calculate the static viscosity, first we calculate the stress autocorrelation function (SACF)

$$
C_{\eta}(t)=\left\langle P_{x y}(t) P_{x y}(0)\right\rangle
$$

where $P_{x y}(t)$ is the shearing stress

$$
P_{x y}(t)=\sum_{i=1}^{N}\left[m v_{i x} v_{i y}-\frac{1}{2} \sum_{j \neq i}^{N} \frac{x_{i j} y_{i j}}{r_{i j}} \frac{\partial \phi\left(r_{i j}\right)}{\partial r_{i j}}\right]
$$

where $i$ and $j$ are indices for different particles, $N$ is the total number of particles of mass $m, \mathbf{r}_{i}=\left(x_{i}, y_{i}\right)$ is the position of particle $i, x_{i j}=x_{i}-x_{j}, y_{i j}=y_{i}-y_{j}, r_{i j}=$ $\left|\mathbf{r}_{i}-\mathbf{r}_{j}\right|$, and $\phi\left(r_{i j}\right)$ is the interparticle potential. Second, we calculate the static viscosity $\eta$ from the Green-Kubo relation [31],

$$
\eta=\frac{1}{V k T} \int_{0}^{\infty} C_{\eta}(t) d t
$$

Here, $V$ is the simulation volume, which is replaced by the area of the simulation box for $2 \mathrm{D}$ simulations like those reported here.

The Green-Kubo relation, Eq. (4), is intended for use in equilibrium systems, but in this paper we will assess whether it can also be used in systems with a modest level of gas friction as in our experiment 20]. The dust particles in an experiment experience gas friction, in addition to collisions amongst themselves, whereas only the latter are modeled in the Green-Kubo relation. We will carry out simulations, with and without friction, and verify that Eq. (4) yields the same result in both cases.

\section{B. Wave-number-dependent viscosity $\eta(k)$}

The wave-number-dependent viscosity $\eta(k)$ characterizes viscous effects at different length scales. A method of calculating $\eta(k)$ from the trajectories of random motion of molecules in liquids has been developed 39, 40. In calculating $\eta(k)$ using this method, one starts with particle trajectories, such as $x_{i}(t)$ and the perpendicular velocity $v_{i y}(t)$ for the $i$ th particle. These are used to calculate the transverse current, $j_{y}(k, t)=\sum_{i=1}^{N} v_{i y}(t) \exp \left[i k x_{i}(t)\right]$. The normalized transverse current autocorrelation function [39, 40] (TCAF) is then calculated as

$$
C_{T}(k, t)=\left\langle j_{y}^{*}(k, 0) j_{y}(k, t)\right\rangle /\left\langle j_{y}^{*}(k, 0) j_{y}(k, 0)\right\rangle,
$$

where the wave vector $k$ is parallel to the $x$ axis. (Here, $k$ serves only as a Fourier transform variable, and is not intended to characterize any waves.) The wavenumber-dependent viscosity of frictionless systems is calculated [39, 40] using

$$
\eta(k) / \rho=1 /\left(\Phi k^{2}\right),
$$

where $\Phi$ is a time integral representing the area under the TCAF after normalizing the TCAF to have a value of unity at $t=0$. Generally, $\eta(k)$ diminishes gradually as $k$ increases, meaning that viscous effects gradually diminish at shorter length scales.

In [20], we generalized this expression as

$$
\eta(k) / \rho=\left[(1 / \Phi)-\nu_{f}\right] / k^{2}
$$

to account for the friction of gas drag $\nu_{f}$ acting on dust particles. As in Eq.(6), the integral $\Phi$ is a function of $k$. Our derivation of Eq. (7) was provided in the supplementary material of [20]. In this paper, we will carry out simulation tests to validate the use of Eq. (7) for a wide range of $k$. This validation test will be performed for the modest level of gas friction $\nu_{f}$ in our experiment [20].

The TCAF measures the memory of transverse current, which reflects the decay of microscopic velocity shear. The shear decay can be caused by several mechanism in 2D dusty plasma clouds, such as Coulomb collisions amongst dust particles and the friction due to gas drag. We will study how gas friction affects the TCAF later.

\section{SIMULATION METHODS}

In order to test the effects of gas friction, we will compare the results of two simulations: a Langevin MD simulation with friction, and a frictionless equilibrium MD simulation.

Our two simulation methods are the same in many respects. Both use a binary interparticle interaction with a Yukawa pair potential. In both simulations, particles are only allowed to move in a single $2 \mathrm{D}$ plane. Conditions remained steady during each simulation run. For both simulations, the parameters we used were $N=4096$ particles in a rectangular box with periodic boundary conditions. The box had sides $64.1 b \times 55.5 b$. The integration time step was $0.019 \omega_{p d}^{-1}$, and simulation data were recorded for a time duration of $68000 \omega_{p d}^{-1}$ after a steady state was reached. Both of our simulations were performed at 
$\Gamma=68$ and $\kappa=0.5$, which are the same values as in our experiment [20].

Our Langevin MD simulation takes into account the dissipation due to gas friction. The equation of motion that is integrated in the Langevin simulation is 36, 43, 4953

$$
m \ddot{\mathbf{r}}_{i}=-\nabla \sum \phi_{i j}-\nu_{f} m \dot{\mathbf{r}}_{i}+\zeta_{i}(t),
$$

where $\nu_{f} m \dot{\mathbf{r}}_{i}$ is a frictional drag and $\zeta_{i}(t)$ is a random force. There is no thermostat to adjust the temperature; instead the temperature is established by choosing the magnitude of $\zeta_{i}(t)$. Here, we chose the experimental value $\nu_{f}=0.08 \omega_{p d}$ [20]. Note that this gas friction level is modest, i.e., the dust particle motion is underdamped, since $\nu_{f} \ll \omega_{p d}$.

Our frictionless equilibrium MD simulation [43, 50, 54 has no gas friction in the equation of motion

$$
m \ddot{\mathbf{r}}_{i}=-\nabla \sum \phi_{i j}
$$

A Nosé-Hoover thermostat is applied to maintain a desired temperature [50, 54].

Trajectories $\mathbf{r}_{i}(t)$ are found by integrating Eq. (8) or (9) for all particles. An example is shown in Fig. 1 from the frictionless MD simulation.

\section{RESULTS}

\section{A. Hydrodynamic and viscoelastic regimes}

Comparing the results from the two simulations, Fig. 2, we can see how friction speeds the loss of memory of the system's microscopic shearing motion. The memory of the shearing motion is indicated by the decay of the TCAF.

As expected [20], in the typical hydrodynamic limit of long length scales, as shown in Fig. 2(a), the TCAF is just a monotonic decay from unity to zero without any oscillations [20]. We find that at the same hydrodynamic length scale, the TCAF decays much faster with friction than without, indicating that in experimental dusty plasmas gas friction plays an important role in shear decay in large length scales.

When the wave number $k$ is slightly larger, in the intermediate regime between the hydrodynamic and viscoelastic regimes, Fig. 2(b), the difference in TCAF between frictional and frictionless is smaller. The integral of the frictional TCAF is about a half of that for the frictionless TCAF, as seen in the inset of Fig. 2(b). This integral corresponds to $\Phi$, as in Eq. (6) or Eq. (7).

When the wave number $k$ is even larger, in the viscoelastic regime, the TCAF oscillates around zero after its decay due to the elastic effects, Fig. 2(c). In this viscoelastic regime, there is little difference between the TCAF from the two simulations, indicating that at smaller length scales, gas friction does not contribute much to shear decay. The friction plays a larger role in TCAF at larger length scales than smaller length scales.

The calculation of $\eta(k)$ using Eq. (6) or (7) requires choosing an upper limit in the time integral of TCAF $C_{T}(k, t)$. An infinite time is of course impractical for both experiments and simulations, so for a finite value we chose $t_{I}$, the time of the first upward zero crossing of the TCAF [20], as shown in Fig. 2(c). This choice is suitable for two reasons: first, it is sufficiently long to retain both viscous and elastic effects; second, we found that contributions to the integral after $t_{I}$ are negligible, for a TCAF that is not noisy. The calculation result for $\eta(k)$ is not very sensitive to the chosen upper limit. Extending the limit to a higher value would only cause a limited effect on the value of the integral.

\section{B. Validating the generalized $\eta(k)$ expression}

Results for the wave-number-dependent viscosity $\eta(k)$ are presented in Fig. 3(b) and (c) for both simulations.

We find an agreement in the values of $\eta(k)$ for the frictionless and Langevin simulations. This agreement can be seen by comparing the circles in Fig. 3(b) for the frictionless simulation with Eq. (6), and the triangles in Fig. 3(c) for the Langevin simulation with Eq. (7). There is not only a qualitative agreement in the downward trend as the wave number $k$ increases, but also a quantitative agreement. This quantitative agreement is most easily seen by fitting the calculated $\eta(k)$ to the Padé approximant of 20, 39] and comparing the fit parameters, as indicated in Fig. 3 for the smooth curves.

This agreement leads us to our first chief result: a validation of Eq. (7) for computing $\eta(k)$ in the presence of gas friction. Since the two simulations were performed for the same values of $\Gamma$ and $\kappa$, an agreement indicates that Eq. (7) is valid. If there had been a discrepancy between the circles in Fig. 3(b) and the triangles in Fig. 3(c), we would question whether Eq. (77) is valid. We gain confidence in the validity of Eq. (7) by the lack of any significant discrepancy in the two results.

The importance of correcting for friction, in Eq. (7), is demonstrated in Fig. 3(c). If we use Eq. (6) instead, the presence of friction leads to an exaggerated value for $\eta(k)$, as seen by comparing the two sets of data in Fig. 3(c). This exaggeration is most extreme at small wave numbers (where the effect of friction is greatest, as we found in Sec. IV A for the TCAF). 


\section{Testing the Green-Kubo relation for static viscosity in the presence of friction}

To determine whether the Green-Kubo relation, Eq. (4), still provides an accurate calculation of static viscosity $\eta$ of a $2 \mathrm{D}$ Yukawa liquid, in the presence of a modest level of gas friction, we performed a test of Eq. (4) comparing $\eta$ computed from our frictional Langevin simulation and our frictionless simulation. These results for the normalized kinematic static viscosity are $\eta / \rho=$ $(0.26 \pm 0.02) a^{2} \omega_{p d}$ for the frictionless simulation, and $\eta / \rho=(0.27 \pm 0.02) a^{2} \omega_{p d}$ for the Langevin simulation with friction. These values are also shown in Fig. 3(b) and (c) as star symbols. Noting that these results are in agreement within the uncertainty, we conclude that the Green-Kubo relation remains accurate, at least with a modest level of gas friction, for a 2D Yukawa liquid at the value $\Gamma=68$ and $\kappa=0.5$.

A further confirmation of the accuracy of the GreenKubo relation when used with modest levels of friction can be found by examining our $\eta(k)$ in Fig. 3(c). We note an agreement of $\eta(k)$ as $k \rightarrow 0$ with $\eta$ from the GreenKubo relation. This agreement is significant because $\eta(k)$ is computed from the TCAF, which is unrelated to the Green-Kubo relation used to compute $\eta$.

We can provide two intuitive suggestions to explain the accuracy of the Green-Kubo relation in the presence of a modest level of gas friction. First, we note that the gas friction that we have considered is so small that $\nu_{f} / \omega_{p d}<0.1$. This inequality demonstrates that frictional effects will in general be much smaller than effects arising from particle charge as measured by $\omega_{p d}$. Second, the TCAF in Fig. 2 showed us that gas friction has the least effect on motion at small length scales, and dynamical information at these small length scales are also reflected in the Green-Kubo relation because it is based only on fluctuations of individual particle motion.

We cannot rule out the possibility that friction will affect the static viscosity computed using the Green-Kubo relation in other parameter regimes. In fact, for a $3 \mathrm{D}$ Yukawa Langevin simulation at a much lower $\Gamma=2$, Ramazanov and Dzhumagulova found that $\eta$ computed using the Green-Kubo relation diminishes as the friction was raised to a very high level [55].

\section{SUMMARY}

Motivated by experiments with 2D clouds of charged dust particles suspended in a plasma, we carried out two types of simulations, with and without gas friction. We validated the newly-introduced Eq. (7) for calculating $\eta(k)$ as a measure of viscoelasticity, in the presence of gas friction. We also verified that the Green-Kubo relation can accurately measure the static viscosity $\eta$ of the $2 \mathrm{D}$ collection of charged dust particles even when they experience gas friction. The level of gas friction we considered was at a low level $\nu_{f} / \omega_{p d}<0.1$, and the coupling was moderate with $\Gamma=68$ and $\kappa=0.5$, both as in our recent experiment [20].

This work was supported by NSF and NASA.

* Electronic address: yan-feng@uiowa.edu

[1] S. Ichimaru, Rev. Mod. Phys. 54, 1017 (1982).

[2] V.E. Fortov, I.T. Iakubov, and A.G. Khrapak, Physics of Strongly Coupled Plasma (Clarendon Press, Oxford, 2006).

[3] P. Vieillefosse and J.P. Hansen, Phys. Rev. A 12, 1106 (1975).

[4] Z. Donkó, G.J. Kalman, and K.I. Golden, Phys. Rev. Lett. 88, 225001 (2002).

[5] C.-W. Io and L. I, Phys. Rev. E 80, 036401 (2009).

[6] P.K. Shukla and A.A. Mamun, Introduction to Dusty Plasma Physics (Institute of Physics Publishing, Bristol and Philadelphia, 2002).

[7] A. Piel, Plasma Physics (Springer, Heidelberg, 2010).

[8] G.E. Morfill and A.V. Ivlev, Rev. Mod. Phys. 81, 1353 (2009).

[9] Y. Feng, J. Goree, and B. Liu, Phys. Rev. Lett. 100, 205007 (2008).

[10] R.L. Merlino and J.A. Goree, Phys. Today 57(7), 32 (2004).

[11] J.H. Chu and L. I, Phys. Rev. Lett. 72, 4009 (1994).

[12] A. Gavrikov, I. Shakhova, A. Ivanov, O. Petrov, N. Vorona, and V. Fortov, Phys. Lett. A 336, 378 (2005).

[13] A. Melzer, A. Homann, and A. Piel, Phys. Rev. E 53, 2757 (1996).

[14] S. Nunomura, D. Samsonov, and J. Goree, Phys. Rev. Lett. 84, 5141 (2000).

[15] X. Wang, A. Bhattacharjee, and S. Hu, Phys. Rev. Lett. 86, 2569 (2001).

[16] V. Nosenko and J. Goree, Phys. Rev. Lett. 93, 155004 (2004).

[17] U. Konopka, G. E. Morfill, and L. Ratke, Phys. Rev. Lett. 84, 891 (2000).

[18] Y. Feng, J. Goree, and B. Liu, Rev. Sci. Instrum. 78, 053704 (2007).

[19] T.M. Flanagan and J. Goree, Phys. Rev. E 80, 046402 (2009).

[20] Y. Feng, J. Goree, and B. Liu, Phys. Rev. Lett. 105, 025002 (2010).

[21] Y. Feng, J. Goree, and B. Liu, Phys. Rev. Lett. 104, $165003(2010)$

[22] W.-T. Juan, M.-H. Chen, and L. I, Phys. Rev. E 64, 016402 (2001).

[23] M. Wolter and A. Melzer, Phys. Rev. E 71, 036414 (2005).

[24] V. Nosenko, J. Goree, and A. Piel, Phys. Plasmas 13, 032106 (2006).

[25] H.M. Thomas and G.E. Morfill, Nature (London) 379, 806 (1996).

[26] T.E. Sheridan, Phys. Plasmas 15, 103702 (2008).

[27] D. Samsonov, S.K. Zhdanov, R.A. Quinn, S.I. Popel, and G.E. Morfill, Phys. Rev. Lett. 92, 255004 (2004).

[28] A. Einstein, Ann. Phys. 324, 289 (1906).

[29] A. Einstein, Ann. Phys. 339, 591 (1911). 
[30] T. Saigo and S. Hamaguchi, Phys. Plasmas 9, 1210 (2002).

[31] B. Liu and J. Goree, Phys. Rev. Lett. 94, 185002 (2005).

[32] Z. Donkó, J. Goree, P. Hartmann, and K. Kutasi, Phys. Rev. Lett. 96, 145003 (2006).

[33] Z. Donkó and P. Hartmann, Phys. Rev. E 78, 026408 (2008).

[34] Z. Donkó, J. Goree, P. Hartmann, and B. Liu, Phys. Rev. E 79, 026401 (2009).

[35] R. Lakes, Viscoelastic Materials (Cambridge University Press, Cambridge, England, 2009), 1st ed.

[36] S. Ratynskaia, G. Regnoli, K. Rypdal, B. Klumov, and G. Morfill, Phys. Rev. E 80, 046404 (2009).

[37] U. Balucani, R. Vallauri, and T. Gaskell, Phys. Rev. A 35, 4263 (1987).

[38] B. J. Palmer, Phys. Rev. E 49, 359 (1994).

[39] U. Balucani, J. P. Brodholt, P. Jedlovszky, and R. Vallauri, Phys. Rev. E 62, 2971 (2000).

[40] Z. Hu and C. J. Margulis, J. Phys. Chem. B 111, 4705 (2007).

[41] P.K. Kaw and A. Sen, Phys. Plasmas 5, 3552 (1998).

[42] M.S. Murillo, Phys. Plasmas 7, 33 (2000).

[43] Z. Donkó, J. Goree, and P. Hartmann, Phys. Rev. E 81, 056404 (2010).
[44] S. Ratynskaia, K. Rypdal, C. Knapek, S. Khrapak, A.V. Milovanov, A. Ivlev, J.J. Rasmussen, and G.E. Morfill, Phys. Rev. Lett. 96, 105010 (2006).

[45] C. L. Chan and L. I, Phys. Rev. Lett. 98, 105002 (2007).

[46] G.J. Kalman, P. Hartmann, Z. Donkó, and M. Rosenberg, Phys. Rev. Lett. 92, 065001 (2004).

[47] D.C. Montgomery and D.A. Tidman, Plasma Kinetic Theory (McGraw-Hill, New York, 1964).

[48] R.K. Pathria, Statistical Mechanics (Pergamon Press, Oxford, 1972).

[49] Y. Feng, B. Liu, and J. Goree, Phys. Rev. E 78, 026415 (2008).

[50] Y. Feng, J. Goree, and B. Liu, Phys. Rev. E 82, 036403 (2010).

[51] L.-J. Hou, A. Piel, and P.K. Shukla, Phys. Rev. Lett. 102, 085002 (2009).

[52] T. Ott and M. Bonitz, Phys. Rev. Lett. 103, 195001 (2009).

[53] O.S. Vaulina, E.A. Lisin, A.V. Gavrikov, O.F. Petrov, and V.E. Fortov, Phys. Rev. Lett. 103, 035003 (2009).

[54] B. Liu and J. Goree, Phys. Rev. E 75, 016405 (2007).

[55] T.S. Ramazanov and K.N. Dzhumagulova, Contrib. Plasma Phys. 48, 357 (2008). 
FIG. 1: (color online). Trajectories of particles from the frictionless MD simulation. Similar trajectories for the experiment and a Langevin MD simulation were reported in [20]. All simulation results here are for $\Gamma=68, \kappa=0.5$.

FIG. 2: (color online). Transverse current autocorrelation functions (TCAF) from simulations in different regimes: (a) hydrodynamic, (b) intermediate between hydrodynamic and viscoelastic, and (c) viscoelastic. Friction plays a larger role at smaller $k$, i.e., longer length scales. After a long time, the TCAF always decays to zero. (For the small $k$ case without friction (a), the TCAF approaches zero after a great time, longer than shown here.) The inset in (b) is the cumulative time integral of TCAF.

FIG. 3: (color online). Wave-number-dependent viscosity $\eta(k)$ from (a) the experiment, (b) the Langevin MD simulation, and (c) the frictionless MD simulation. The agreement of the smooth curves in (b) and (c) validates Eq. (7) for calculating $\eta(k)$ in the presence of gas friction. Using Eq. (6) in the presence of gas friction (circle data points in (c)) would lead to an exaggerated value for $\eta(k)$, especially at longer length scales. Also shown in (b) and (c) is the calculated static viscosity $\eta$ based on the Green-Kubo relation as indicated with star symbols. Panel (a) is reprinted from [20]. 